\title{
Assaying proline hydroxylation in recombinant collagen variants by liquid chromatography-mass spectrometry
}

\author{
S W Polly Chan ${ }^{1}$, John Greaves ${ }^{2}$, Nancy A Da Silva ${ }^{1 *}$ and Szu-Wen Wang ${ }^{1 *}$
}

\begin{abstract}
Background: The fabrication of recombinant collagen and its prescribed variants has enormous potential in tissue regeneration, cell-matrix interaction investigations, and fundamental biochemical and biophysical studies of the extracellular matrix. Recombinant expression requires proline hydroxylation, a post-translational modification which is critical for imparting stability and structure. However, these modifications are not native to typical bacterial or yeast expression systems. Furthermore, detection of low levels of 4-hydroxyproline is challenging with respect to selectivity and sensitivity.

Results: We have developed a new liquid chromatography-mass spectrometry (LC-MS) method to evaluate proline hydroxylation in recombinant collagen. This assay was tested in different Saccharomyces cerevisiae expression systems to evaluate the effect of gene ratio between prolyl-4-hydroxylase and collagen on the extent of hydroxylation. These systems used a human collagen III gene that was synthesized de novo from oligonucleotides. The LC-MS assay does not require derivatization, uses only picomoles of sample, and can measure proline hydroxylation levels in recombinant and native collagen ranging from approximately $0 \%$ to $40 \%$. The hydroxylation values obtained by LC-MS are as accurate and as precise as those obtained with the conventional method of amino acid analysis.

Conclusions: A facile, derivatization-free LC-MS method was developed that accurately determines the percentage of proline hydroxylation in different yeast expression systems. Using this assay, we determined that systems with a higher collagen-to-hydroxylase gene copy ratio yielded a lower percentage of hydroxylation, suggesting that a specifically balanced gene ratio is required to obtain higher hydroxylation levels.
\end{abstract}

Keywords: Hydroxyproline, Liquid chromatography-mass spectrometry, LC-MS assay, Recombinant collagen

\section{Background}

Collagen is the most abundant class of proteins in the extracellular matrix and it demonstrates dynamic interactions with its biological microenvironment [1]. Therefore, fabrication of de novo, synthetic collagen in which specific sequences can be prescribed has tremendous potential in tissue engineering, drug delivery applications, and fundamental biological studies of the extracellular matrix. Towards this end, two challenges exist: (1) the synthesis of genes encoding the collagenmimetic polymers which contain repeating amino acid

\footnotetext{
*Correspondence: ndasilva@uci.edu; wangsw@uci.edu

${ }^{1}$ Department of Chemical Engineering and Materials Science, University of California, Irvine, CA 92697, USA

Full list of author information is available at the end of the article
}

sequences, and (2) the hydroxylation of prolines in a recombinant system. To address the first constraint, our research group has developed a platform that yields genes encoding for full-length, modular collagen and its variants. This approach allows mixing-and-matching of specific functional amino acid sequences from the different families of collagens and enables introduction of non-native sequences at defined locations, combinations, and number of occurrences [2].

We are addressing the second challenge, recombinant expression coupled with post-translational proline hydroxylation, by designing and optimizing genetic modifications in Saccharomyces cerevisiae. Proline hydroxylation is particularly important, as it imparts stability and structure to collagen. However, 
bacterial and yeast expression systems do not natively perform this post-translational modification, and must be engineered to produce prolyl-4-hydroxylase ( $\alpha$ and $\beta$ subunits). These systems, however, tend to yield biopolymers with lower levels of proline hydroxylation, ranging from $0.5 \%$ to $38 \%$ proline hydroxylation in recombinant collagens produced in $S$. cerevisiae [2-4] and $44.2-47.2 \%$ in the best $P$. pastoris reported systems [5,6]. In comparison, fibrillar human collagens from native tissues show $42-54 \%$ hydroxylation $[7,8]$.

Given the large possible range of values, we needed an accessible and facile assay that can determine the level of proline hydroxylation in future libraries of recombinant collagen and its variants. Such an assay should also use relatively small amounts (pmol) of sample, require minimal processing and derivatization, and potentially enable high-throughput scaleup. As others have noted, however, detection of 4hydroxyproline (HYP) is particularly challenging with respect to both selectivity and sensitivity [9].

To address these difficulties, analytical methods for HYP often require derivatization [10-13]. In fact, the conventional method of determining the percentage of proline hydroxylation, amino acid analysis (AAA), measures the concentration of amino acid residues after derivatization with a fluorescent probe, such as ninhydrin $[14,15]$. However, to assay relatively small quantities (picomole), a sensitive and expensive fluorescence detector is required on the liquid chromatography system. Protocols using radioisotopes have also been developed [16], but the logistics of using radioactive compounds are inconvenient if appropriate research infrastructure is not in place.

Our aim was to develop a rapid method to quantify HYP without further derivatization by utilizing mass spectrometry (MS) instrumentation that would be accessible in most research institutions. Mass spectrometry protocols requiring no additional chemical reaction have been reported using hydrophilic interaction chromatography (HILIC) [13] and tandem (LC-MS/MS) mass-spectrometry with multiple reaction monitoring (MRM) [9,17]. Our method to quantify the amounts of proline (PRO) and HYP in different collagen samples uses a simple and standard reversed-phase liquid chromatograph coupled to a single analyzer time-of-flight MS (LC-MS) and requires no sample derivatization.

We applied this LC-MS assay to engineered S. cerevisiae systems that we expected would yield various levels of proline hydroxylation. These yeast strains contained different collagen to prolyl-4-hydroxylase gene ratios on plasmid vectors. To determine the reliability of this LC-MS assay, these hydroxylation results were compared to conventional AAA.

\section{Methods}

Quantification of proline and hydroxyproline

Liquid Chromatography - Mass Spectrometry (LC-MS) method

The LC-MS consisted of an Agilent 1100 instrument and a Waters LCT Classic mass spectrometer in an open access user facility. The liquid chromatography separations used a solvent system of $2 \%$ acetonitrile and $0.2 \%$ acetic acid in water (solvent $\mathrm{A}$ ) and $0.2 \%$ acetic acid in acetonitrile (solvent B), with a 45-minute solvent program that reached $25 \% \mathrm{~B}$ at $25 \mathrm{~min}$ followed by a rapid ramp to $95 \%$ B to remove unwanted compounds from the column. Ten- $\mu$ l samples dissolved in acetonitrile/ water $(50: 50 \mathrm{v} / \mathrm{v})$ were injected onto a Phenonenex Luna $5 \mu \mathrm{C} 18100 \AA 150 \mathrm{~mm}$ long $\times 2.0 \mathrm{~mm}$ internal diameter column connected directly to the mass spectrometer. Electrospray ionization (ESI) was used in positive ion mode.

\section{Determination of standard curves}

Calibration standards were D-proline (Aldrich) and trans-4-hydroxy-L-proline (Aldrich), and the internal standard (IS) was glycyl phenylalanine (Sigma). Dproline could be used in place of L-proline because the two stereoisomers give identical elution times and calibration curves. To obtain calibration curves, we injected different concentrations of PRO and HYP which were dissolved in acetonitrile/water (50:50 v/v) containing $0.5 \mu \mathrm{g} / \mathrm{ml}$ glycyl phenylalanine. The PRO, HYP, and IS peaks were identified based on their masses and retention times. Reconstructed ion chromatograms (RIC) for the protonated PRO and HYP and major fragments were plotted. The internal standard RIC included the molecular species, its fragments, and the acetonitrile adduct ions. Calibration curves were obtained by plotting the area ratios of the PRO/IS and HYP/IS against the PRO and HYP concentrations. Three sets of calibration curves were determined immediately before and/or after each set of collagen samples was run on the LC-MS, and an average was taken of the linear regression equations. Linearity was acceptable $\left(\mathrm{R}^{2}\right.$ greater than 0.990$)$ for PRO concentrations between $0.2 \mu \mathrm{g} / \mathrm{ml}$ and $5 \mu \mathrm{g} / \mathrm{ml}$ and for HYP concentrations between $0.2 \mu \mathrm{g} / \mathrm{ml}$ and $1.5 \mu \mathrm{g} / \mathrm{ml}$. A secondary confirmation was obtained by diluting analytical samples by a factor of two and ensuring that the resulting concentrations were similarly halved.

\section{Vector and strain constructions}

Haploid Saccharomyces cerevisiae BY4741- $\triangle$ TRP1 (strain BY4741 with a TRP1 deletion: MATa his $3 \Delta 1$ leu2 $\Delta 0$ met15 $\Delta 0$ ura3 $\Delta 0 \Delta \operatorname{trp} 1:: \operatorname{KanMX}$ ) [18] was used to express the modular collagen and the human prolyl4-hydroxylase. E. coli strain XL1-Blue (Stratagene) was used for plasmid maintenance and amplification. Human 
prolyl-4-hydroxylase subunit genes $\mathrm{p} 4 \mathrm{Ha}$ and $\mathrm{p} 4 \mathrm{Hb}$ were cloned into a low copy CEN/ARS vector and cotransformed with the $2 \mu$ modular collagen plasmid into BY4741- $\triangle$ TRP1 as described previously [19]. The p4Ha gene was cloned under the GAL10 promoter and the $\mathrm{p} 4 \mathrm{Hb}$ gene with a yeast invertase (SUC2) leader sequence was cloned under the GAL1 promoter on the same CEN/ARS plasmid. The modular collagen gene was under the control of the S. cerevisiae GAL1 promoter [2]. To test the effect of balancing the collagento-hydroxylase gene copy ratio, the collagen gene was placed on a CEN/ARS plasmid. The GAL1p-MCOL gene cassette was excised using HindIII from YEpMCOL [2], inserted into YCplac22 [20] to create YCpMCOLtrp, a low-copy CEN/ARS vector. This vector was cotransformed with the CEN/ARS hydroxylase vector into BY4741- $\triangle T R P 1$. As a negative control strain (no hydroxylase present), YEpMCOL was transformed into BY4741- $\triangle$ TRP1 alone without the hydroxylase plasmid.

\section{Collagen expression and purification}

Strains carrying the dual plasmids were inoculated into selective medium and cultured as described in Chan et al. [2]. Cells were lysed and their background protein was digested with pepsin. Recombinant collagen was purified from the digested protein by cationic exchange chromatography as described in Chan et al. [2].

\section{Preparation and analysis of FPLC-purified collagen samples}

Samples of dried bovine collagen ( 5 to $12 \mu \mathrm{g}$; Millipore) and FPLC-purified recombinant collagen samples (40 to $80 \mu \mathrm{g})$ were hydrolyzed in $6 \mathrm{~N}$ hydrochloric acid (Pierce) vapor at $150^{\circ} \mathrm{C}$ for 90 minutes under vacuum [15]. The hydrolysate was resuspended with acetonitrile/water (50:50 v/v) containing $0.5 \mu \mathrm{g} / \mathrm{ml}$ of the IS and injected onto the LC-MS system. For comparison, AAA was performed by the Biopolymer Lab at UCLA (http://www. mbi.ucla.edu/services/biopolymer-laboratory). For AAA, protein samples were hydrolyzed in $6 \mathrm{~N}$ (vapor-phase) $\mathrm{HCl}$ for 22 hours at $110^{\circ} \mathrm{C}$. The free amino acids were then derivatized with 6-aminoquinoly- $\mathrm{N}$-hydroxysuccinimidyl carbamate (AQC, Waters), a fluorescent aminoreactive probe, and the products were fractionated and quantified by reversed-phase HPLC.

\section{Preparation and analysis of SDS-PAGE-purified collagen samples}

To investigate the feasibility of isolating collagen from SDS-PAGE for analysis, SDS-PAGE loading buffer $(6 \times)$ was added to collagen samples (totalling $320 \mu \mathrm{l}$ ), heated at $95^{\circ} \mathrm{C}$ for 5 minutes, and run on a $7 \%$ SDS-PAGE gel. The gel was washed with water, stained in $0.1 \%$ Coomassie Blue R-250 (Fisher) dissolved in $10 \%$ acetic acid $/ 50 \%$ methanol $/ 40 \%$ water, and then destained in $10 \%$ acetic acid $/ 50 \%$ methanol $/ 40 \%$ water to determine band locations. The collagen bands were excised, placed in a glass vial, further destained by washing three times in 10\% acetic acid $/ 50 \%$ methanol $/ 40 \%$ water, and stored at $4^{\circ} \mathrm{C}$ overnight. To extract the collagen, acetonitrile (Fisher, Optima LC-MS grade, $100 \mu \mathrm{l}$ ) was added to the vial that was then sonicated in a water bath for 20 minutes at $40 \mathrm{kHz}$. The acetonitrile was recovered and a second extraction with $50 \mu$ l fresh acetonitrile was performed. The combined acetonitrile extracts were dried under nitrogen in a LC-MS vial and then subjected to hydrolysis as described for FPLC-purified samples. The products were resuspended with $0.1 \mathrm{ml}$ acetonitrile:water $(50: 50 \mathrm{v} / \mathrm{v})$ containing $0.5 \mu \mathrm{g} / \mathrm{ml}$ IS and analyzed by LC-MS.

Table 1 Percent hydroxylation of proline in bovine collagen III and in recombinant human collagen III

\begin{tabular}{lll}
\hline & LC-MS Assay (\%) & Amino Acid Analysis (\%) \\
\hline Bovine collagen III (as-received) & $38 \pm 1.1$ & $37 \pm 2.7$ \\
Bovine collagen III (extracted from SDS-PAGE) & $36 \pm 3.4$ & N.a \\
Recombinant human collagen III & N.D.* & \\
$\quad$ No hydroxylase (p4Ha, p4Hb) genes & & $5.2 \pm 1.3$ \\
$\quad$ Collagen on 2-micron plasmid & $5.8 \pm 0.5$ & $12^{\S}$ \\
Recombinant human collagen III & & $12 \pm 1.8$ \\
$\quad \begin{array}{l}\text { CEN/ARS-p4Ha-p4Hb with SUC2 signal } \\
\text { Collagen on 2-micron plasmid }\end{array}$ & & \\
Recombinant human collagen III & & \\
CEN/ARS-p4Ha-p4Hb with SUC2 signal \\
Collagen on CEN/ARS plasmid
\end{tabular}

* "N.D." denotes no significant signal of hydroxyproline above background noise.

§only 1 AAA result available. 


\section{Percentage hydroxylation calculation}

The HYP present in collagen is the product of in vivo hydroxylation of PRO residues [21], and we defined the percent hydroxylation of proline to be (moles of HYP)/ (moles of HYP + moles of PRO) $\times 100 \%$. The percent hydroxylation was calculated using concentrations obtained from the LC-MS data. Final values presented in Table 1 are the result of averaging triplicate measurements from 2-3 independent experiments for LC-MS, and measurements of triplicate experiments for AAA. The reported percent hydroxylation values obtained by AAA have the background chemical noise $(0.18 \%)$ subtracted; this was obtained from the yeast strain containing the collagen gene, but without hydroxylase genes (negative control). The background signal observed in the LC-MS for this negative control was less than $1 \%$ relative to readings of any of the HYP standards (Figure 1) and was therefore considered insignificant.

\section{Results and Discussions}

\section{LC-MS method quantifies proline and hydroxyproline}

The peaks for proline (PRO), hydroxyproline (HYP), and the internal standard (IS) were identified from injecting pure samples in the LC-MS system. The choice of the internal standard was based on stability, similarity of chemical nature, molecular mass (when compared with PRO and HYP), and commercial availability. Elution times were 1.6 minutes for HYP, 1.65 minutes for PRO, and 2.25 minutes for the IS, glycyl phenylalanine. These elution times were expected for non-derivatized amino acids on the reversed-phase column. The peak area ratios between the fixed amount of internal standard and the two amino acids, proline and hydroxyproline, yielded calibration curves that increased linearly with the amino acid concentrations (Figure 1). Typical linear

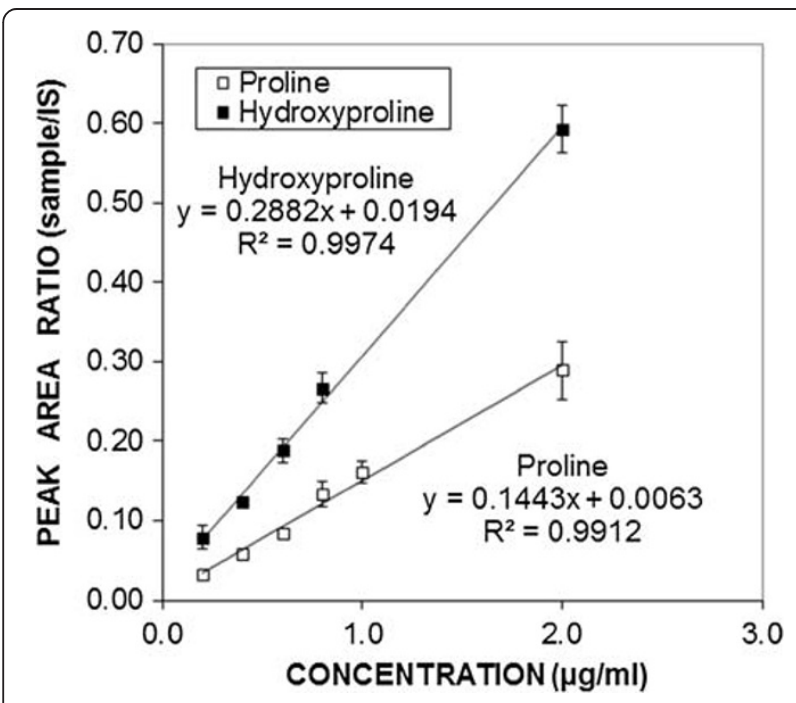

Figure 1 Proline and hydroxyproline standard curves. regression equations were $\mathrm{y}=0.144 \mathrm{x}+0.006$ for $\mathrm{PRO}$ and $y=0.288 x+0.019$ for HYP, with the coefficients of regression $\left(R^{2}\right)$ greater than 0.994. Therefore, for unknown protein hydrolysates, the peak area ratios for the two amino acids against the internal standard can be used to determine the amino acid relative concentrations.

\section{LC-MS assay gives values comparable to conventional amino acid analysis over full range of proline hydroxylation for collagen}

To evaluate our LC-MS method, we examined percent hydroxylation of commercial bovine collagen III and recombinant collagen from two $S$. cerevisiae strains engineered to produce prolyl-4-hydroxylase. From prior amino acid analysis (AAA), we expected that the percent hydroxylation for the recombinant systems would yield intermediate values that are less than those of native (bovine) collagen and therefore enable us to probe the full range of possible hydroxylation values. Figure 2 shows reconstructed ion chromatographs (RIC) of representative collagen samples. Based on the mass and elution times, the PRO, HYP, and IS peaks were identified. The integrated areas under the PRO and HYP peaks were divided by the integrated area of the IS and their relative concentrations were obtained using the respective standard curves. Percentage proline hydroxylation was then determined as described in Methods.

The percentage proline hydroxylation values are summarized in Table 1. For native bovine collagen III (positive control), the percent hydroxylation of proline was determined by our LC-MS assay to be $38 \pm 1.1 \%$ ( $n=3$ separate samples, with each sample injected in triplicate). The percentage hydroxylation of bovine collagen was also determined by amino acid analysis (AAA), which showed $37 \pm 2.7 \%$ hydroxylation. These values are consistent with a previously-reported value of $41 \%$ hydroxylation for bovine collagen as determined by AAA [22]. For the negative control, we placed a collagen gene on a 2-micron plasmid in yeast that did not contain the prolyl hydroxylase genes. As expected, neither the LCMS nor AAA methods yielded hydroxylation signals beyond background chemical noise.

To examine the ability of the LC-MS assay to determine intermediate hydroxylation levels, we evaluated collagen produced from a 2-micron vector (high copy number) and a CEN/ARS (low copy number) vector. In both cases, the hydroxylase enzymes were co-expressed with collagen and carried on a CEN/ARS vector. For these recombinant samples, our LC-MS method obtained percent hydroxylation of $5.8 \pm 0.5 \%$ for collagen on the 2-micron (high copy number) vector and $12 \pm 1.8 \%$ for the CEN/ARS (low copy number) plasmid. In comparison, AAA of these samples gave $5.2 \pm 1.3 \%$ and $12 \%$ hydroxylation, respectively. Collectively, our 


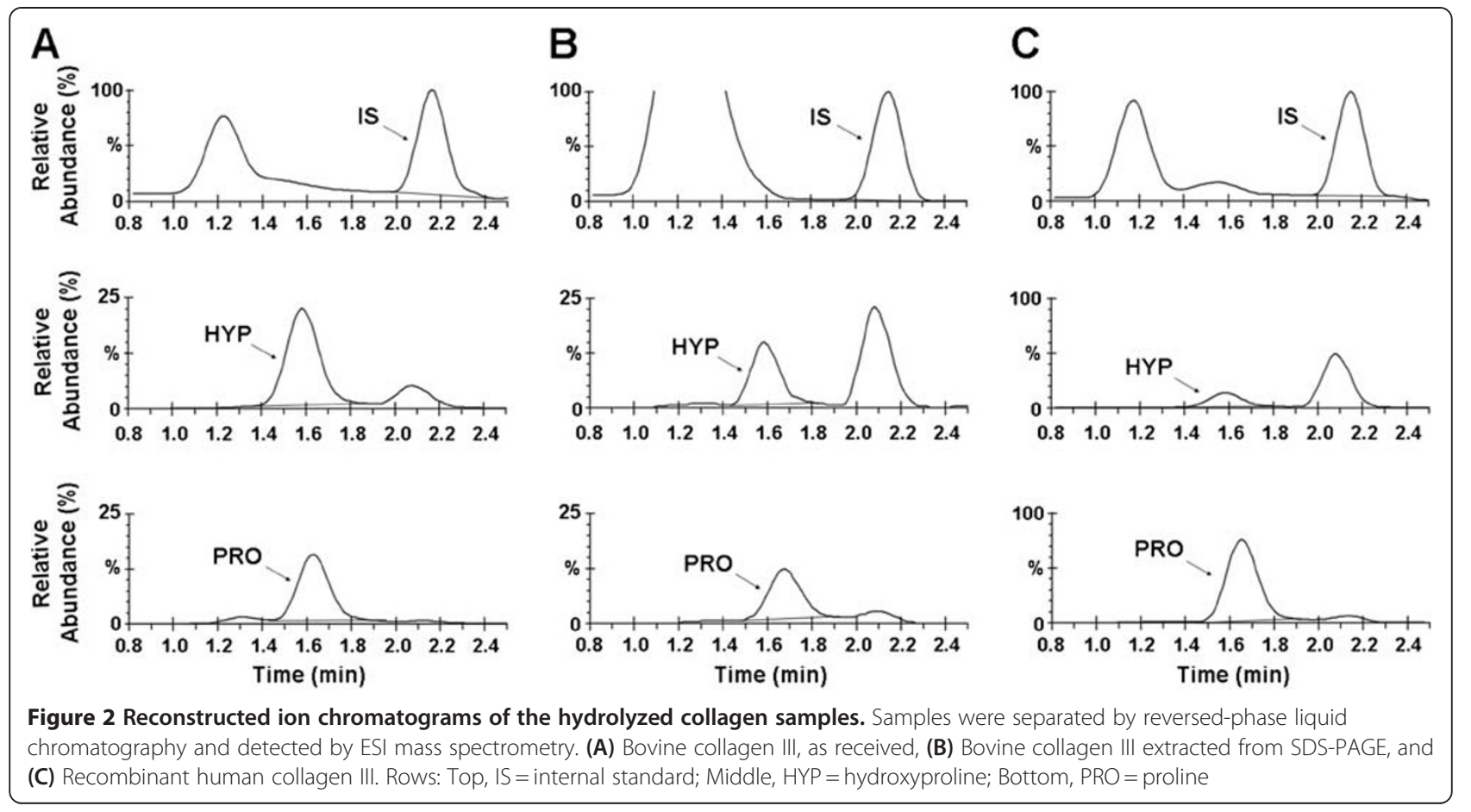

data shows that for determining the percentage of hydroxylated proline, the LC-MS method yields values equivalent to the conventional strategy of AAA.

Furthermore, the precision for the hydroxylation measurements using our LC-MS method for bovine, recombinant (2-micron), and recombinant (CEN/ ARS) collagen samples were $2.9 \%, 7.9 \%$, and $15 \%$ relative standard deviation (RSD), respectively. In contrast, using AAA, the RSD for bovine collagen and recombinant collagen (2-micron) were $7.3 \%$ and $23 \%$, respectively. This demonstrates that the LC-MS method is also at least as precise, if not more so, as conventional AAA.

Many AAA systems have a sensitivity of 1 pmole [15], with a recommended minimum of 50 pmoles for quantitative analysis. Our experience with AAA has shown that this minimum level of 50 pmoles is especially critical for the quantification of HYP. Below this level, the elution time of HYP in AAA is very close to that of the hydrolysis by-product, 6aminoquinoline, resulting in overlapping baselines in the chromatogram. In our LC-MS assay, the lower limit of quantification for the pure amino acids is approximately 15 pmole on column for both PRO and HYP. In addition, because reconstructed ion chromatographs are used, there is no problem of an overlap with 6 -aminoquinoline. Therefore, the amount of samples needed for both our LC-MS assay and AAA are comparable in magnitude, with the LC-MS assay requiring slightly less sample.

\section{Evaluation of SDS-PAGE sample purification method}

The recombinant human collagen variants presented in Table 1 were purified by fast protein liquid chromatography (FPLC), as described in Methods. However, FPLC can be time-consuming and not practical for larger-scale screens of protein variants, so we investigated whether collagen that was extracted from SDS-PAGE could yield reasonable protein hydroxylation values using the LCMS assay. Success in quantifying HYP from these extracts would enable analysis of samples without development of lengthy purification protocols.

Although purified by the commercial source, the bovine collagen still shows impurity bands when run on SDS-PAGE gels. The percent hydroxylation for bovine collagen III extracted from SDS-PAGE gave $36 \pm 3.4 \%$, which was close to the values measured from the asreceived samples $(38 \pm 1.1 \%)$. However, the RSD (an indicator of repeatability) of bovine collagen extracted from SDS-PAGE was 9.4\%, which is higher than the $2.9 \%$ RSD of as-received collagen, suggesting that the additional steps and reagents required in SDS-PAGE extraction may decrease the precision of our method.

For recombinant human collagen extracted from SDSPAGE gels, we found that LC-MS could indeed detect the significantly lower levels of hydroxylation present (relative to fully hydroxylated bovine collagen); however, these results were not as accurate or precise as for FPLC-purified samples. This demonstrates that extraction from SDS-PAGE could potentially be used to screen protein candidates to identify desired levels of hydroxylation, 
but chromatography-based purification may be necessary for in-depth analysis.

\section{Balanced enzyme to collagen gene ratio is required for higher proline hydroxylation levels}

We have assayed the proline hydroxylation levels of recombinant collagen produced in two dual-plasmid yeast strains. The first strain overexpressed the collagen using a $2 \mu$ plasmid and produced a $5.8 \%$ hydroxylated protein. The second strain expressed collagen from a stable, low copy CEN/ARS plasmid, and in this case the hydroxylation increased to $12 \%$. In both cases, the two hydroxylase genes were carried on a second CEN/ARS plasmid. Therefore, the two strains have different collagen to hydroxylase gene ratios, and this is reflected in the percent hydroxylation of the collagen produced. When the copy number of the collagen gene was high relative to the hydroxylase genes, we obtained a lower level of hydroxylation.

Native collagen has a significantly greater proline hydroxylation level (38\% for bovine collagen III) than our systems reported here. Based on the findings from the two dual plasmid systems, the percentage hydroxylation should increase if the hydroxylase and collagen expression levels are appropriately balanced. We expect that further increasing the number of $\mathrm{p} 4 \mathrm{H}$ genes or their expression levels should increase hydroxylation. In light of this, our current work includes integrating the two hydroxylase subunits and the collagen gene cassettes into the yeast genome to control tightly the collagen-toprolyl hydroxylase gene copy ratio. Our facile LC-MS assay will be very helpful in determining the percentage hydroxylation of these integrated strains and identifying the construct with hydroxylation levels closest to native collagen.

\section{Conclusions}

We have developed an LC-MS method for determining the percent hydroxylation of collagen from native and recombinant sources. In our studies, this method has been used to analyze native collagen and recombinant collagen (expressed in yeast) with hydroxylation percentages ranging from 0 to approximately 40\%. Our LC-MS method is as accurate and precise as the conventional amino acid analysis, and has been used to assess hydroxylation levels for different recombinant $S$. cerevisiae strains producing collagen. The results demonstrate the importance of balancing collagen and hydroxylase expression levels in the yeast host. Overall, this new LCMS method is a rapid and reliable analytical method for screening proteins with varying levels of proline hydroxylation, and it provides an alternative method to conventional amino acid analysis.
Competing interests

The authors declare that they have no competing interests.

\section{Authors' contributions}

SWPC performed molecular cloning, design of plasmids, expression and purification of proteins, and design and running LC-MS assays. JG participated in the design of the LC-MS method. NAD and SWW participated in the design and coordination of the overall study. All authors contributed to the writing of the manuscript, and all authors have read and approved the final manuscript.

\section{Acknowledgements}

We gratefully acknowledge Margaret Condron and David Teplow at the UCLA Biopolymer Laboratory core facility for assistance with amino acid analysis. This work was supported by the National Science Foundation (DMR0706669, DMR-1006999, and CBET-1034566).

\section{Author details}

${ }^{1}$ Department of Chemical Engineering and Materials Science, University of California, Irvine, CA 92697, USA. ²Department of Chemistry, University of California, Irvine, CA 92697, USA.

Received: 17 April 2012 Accepted: 13 July 2012

Published: 17 August 2012

\section{References}

1. Gelse K, Poschl E, Aigner T: Collagens - structure, function, and biosynthesis. Adv Drug Deliv Rev 2003, 55(12):1531-1546.

2. Chan SWP, Hung SP, Raman SK, Hatfield GW, Lathrop RH, Da Silva NA, Wang SW: Recombinant human collagen and biomimetic variants using a de novo gene optimized for modular assembly. Biomacromolecules 2010, 11(6):1460-1469.

3. Vaughan PR, Galanis M, Richards KM, Tebb TA, Ramshaw JA, Werkmeister JA: Production of recombinant hydroxylated human type III collagen fragment in Saccharomyces cerevisiae. DNA Cell Biol 1998, 17(6):511-518.

4. Toman PD, Chisholm G, McMullin H, Gieren LM, Olsen DR, Kovach RJ, Leigh $S D$, Fong BE, Chang R, Daniels GA, et al: Production of recombinant human type I procollagen trimers using a four-gene expression system in the yeast Saccharomyces cerevisiae. J Biol Chem 2000, 275(30):2330323309.

5. Vuorela A, Myllyharju J, Nissi R, Pihlajaniemi T, Kivirikko Kl: Assembly of human prolyl 4-hydroxylase and type III collagen in the yeast Pichia pastoris: formation of a stable enzyme tetramer requires coexpression with collagen and assembly of a stable collagen requires coexpression with prolyl 4-hydroxylase. EMBO J 1997, 16(22):6702-6712.

6. Nokelainen M, Tu HM, Vuorela A, Notbohm H, Kivirikko Kl, Myllyharju J: High-level production of human type I collagen in the yeast Pichia pastoris. Yeast 2001, 18(9):797-806.

7. Uitto J: Collagen polymorphism - isolation and partial characterization of alpha-1 (I)-trimer molecules in normal human skin. Arch Biochem Biophys 1979, 192(2):371-379.

8. Chung E, Miller EJ: Collagen polymorphism - characterization of molecules with chain composition [Alpha-1(III)]3 in human tissues. Science 1974, 183(4130):1200-1201.

9. Kindt E, Gueneva-Boucheva K, Rekhter MD, Humphries J, Hallak H: Determination of hydroxyproline in plasma and tissue using electrospray mass spectrometry. J Pharm Biomed Anal 2003, 33(5):1081-1092.

10. Prockop DJ, Udenfriend S: A specific method for the analysis of hydroxyproline in tissues and urine. Anal Biochem 1960, 1(3):228-239.

11. Husek P: Rapid derivatization and gas-chromatographic determination of amino-acids. J Chromatogr 1991, 552(1-2):289-299.

12. Marfey P: Determination of d-amino acids. 2. Use of a bifunctional reagent, 1,5-difluoro-2,4-dinitrobenzene. Carlsberg Res Commun 1984, 49 (6):591-596

13. Langrock T, Czihal P, Hoffmann R: Amino acid analysis by hydrophilic interaction chromatography coupled on-line to electrospray ionization mass spectrometry. Amino Acids 2006, 30(3):291-297.

14. Eastoe JE: Amino acid composition of mammalian collagen and gelatin. Biochem J 1955, 61(4):589-600.

15. Ozols J: Amino acid analysis. In Methods in Enzymology, Volume 182. Edited by Deutscher MP. New York: Academic Press; 1990:587-601. 
16. Juva K, Prockop DJ: Modified procedure for assay of H3-or C14-Labeled hydroxyproline. Anal Biochem 1966, 15(1):77-83.

17. Colgrave $M L$, Allingham $P G$, Jones $A$ : Hydroxyproline quantification for the estimation of collagen in tissue using multiple reaction monitoring mass spectrometry. J Chromatogr A 2008, 1212(1-2):150-153.

18. Winzeler EA, Shoemaker DD, Astromoff A, Liang H, Anderson K, Andre B, Bangham R, Benito R, Boeke JD, Bussey $H$, et al: Functional characterization of the $\mathrm{S}$-cerevisiae genome by gene deletion and parallel analysis. Science 1999, 285(5429):901-906.

19. Chan SWP: Fabrication of modular human collagen and collagen variants in yeast. PhD thesis. University of California, Irvine: Department of Chemical Engineering; 2012.

20. Gietz RD, Sugino A: New yeast-Escherichia-coli shuttle vectors constructed with in vitro mutagenized yeast genes lacking 6-base pair restriction sites. Gene 1988, 74(2):527-534

21. Berg RA: Determination of 3-hydroxyproline and 4-hydroxyproline. Methods Enzymol 1982, 82:372-398.

22. Steven FS, Jackson DS: Purification and amino acid composition of monomeric and polymeric collagens. Biochem J 1967, 104(2):534-539.

doi:10.1186/1472-6750-12-51

Cite this article as: Chan et al: Assaying proline hydroxylation in recombinant collagen variants by liquid chromatography-mass spectrometry. BMC Biotechnology 2012 12:51.

\section{Submit your next manuscript to BioMed Central and take full advantage of:}

- Convenient online submission

- Thorough peer review

- No space constraints or color figure charges

- Immediate publication on acceptance

- Inclusion in PubMed, CAS, Scopus and Google Scholar

- Research which is freely available for redistribution 